\title{
Some Simple Models for Nonlinear Age-Dependent Population Dynamics
}

\author{
MORTON E. GURTIN AND RICHARD C. MACCAMY \\ Department of Mathematics \\ Carnegie-Mellon University \\ Pittsburgh, Pennsylvania 15213
}

Received 23 June 1978; revised 24 August 1978

\begin{abstract}
This paper presents two simple models for nonlinear age-dependent population dynamics. In these models the basic equations of the theory reduce to systems of ordinary differential equations. We discuss certain qualitative aspects of these systems; in particular, we show that for many cases of interest periodic solutions are not possible.
\end{abstract}

\section{BASIC EQUATIONS}

Recently, Gurtin and MacCamy [1] introduced a nonlinear ${ }^{1}$ theory of population dynamics with age dependence. This theory is based on the equations $^{2}$

$$
\begin{aligned}
& \rho_{a}(a, t)+\rho_{t}(a, t)+\mu(a, P(t)) \rho(a, t)=0, \\
& B(t)=\rho(0, t)=\int_{0}^{\infty} \beta(a, P(t)) \rho(a, t) d a, \\
& P(t)=\int_{0}^{\infty} \rho(a, t) d a,
\end{aligned}
$$

where

$\rho(a, t)$ is the age distribution, that is, the number of individuals of age $a$ at time $t(a \geqslant 0, t \geqslant 0)$;

$P(t)$ is the total population;

$B(t)$ is the birth rate;

$\mu(a, P)$ is the survival function;

$\beta(a, P)$ is the maternity function.

'The linear theory is discussed in detail by Hoppensteadt [2].

${ }^{2}$ Subscripts indicate partial differentiation.

MATHEMATICAL BIOSCIENCES 43:199-211 (1979) 
The functions $\mu(a, P)$ and $\beta(a, P)(a \geqslant 0, P \geqslant 0)$ are assumed prescribed, as is the initial age distribution $\varphi$. The corresponding initial-value problem then consists in finding a solution $\rho(a, t)$ of $(1.1)$ which satisfies the initial condition

$$
\rho(a, 0)=\varphi(a) .
$$

The system (1.1), (1.2) can be converted to a pair of nonlinear integral equations, which can be used, under reasonable hypotheses, to establish existence and uniqueness [1]. Here we will show that when $\mu$ and $\beta$ have certain simple forms, (1.1) and (1.2) reduce to an initial-value problem for a nonlinear system of ordinary differential equations.

Before discussing these systems, however, we note that the partial differential equation $(1.1)_{1}$ [with the conditions $\rho(0, t)=B(t), \rho(a, 0)=\varphi(a)$ ] can be integrated along characteristics to give ${ }^{3}$

$$
\begin{aligned}
& \rho(a, t)=\varphi(a-t) \pi(a-t, a, 0), \quad a \geqslant t, \\
& \rho(a, t)=B(t-a) \pi(0, a, t-a), \quad t>a,
\end{aligned}
$$

where

$$
\pi\left(a_{0}, a, t\right)=\exp \left\{-\int_{a_{0}}^{a} \mu\left(\alpha, P\left(\alpha-a_{0}+t\right)\right) d \alpha\right\} .
$$

Thus a knowledge of the quantities $P$ and $B$ will completely determine the age distribution of the population for all time.

\section{MODEL WITH TWO DIFFERENTIAL EQUATIONS}

Before discussing this model we prove the following

\section{LEMMA}

Assume that the survival function $\mu$ is independent of age. Let $\rho$ be a solution of (1.1), and let $g$ be a (sufficiently nice) function of age with ${ }^{4}$

$$
g(a) \rho(a, t) \rightarrow 0 \quad \text { as } \quad a \rightarrow \infty .
$$

Define

$$
\begin{aligned}
& G(t)=\int_{0}^{\infty} g(a) \rho(a, t) d a, \\
& H(t)=\int_{0}^{\infty} g^{\prime}(a) \rho(a, t) d a .
\end{aligned}
$$

${ }^{3}$ Cf. [1], pp. 284-285.

${ }^{4}$ This assumption is reasonable. Indeed, one can show, as a consequence of $(1.1)_{1}$, that $\rho(a, t)$ as a function of $a$ will have compact support at each $t$ provided $\varphi$ has compact support. 
Then

$$
\dot{G}+\mu(P) G-g(0) B=H
$$

Proof. We multiply (1.1), by $g$ and integrate from $a=0$ to $a=\infty$; the result is

$$
\dot{G}(t)+\int_{0}^{\infty} g(a) \rho_{a}(a, t) d a+\mu(P) G(t)=0
$$

and if we integrate the second term by parts and use (2.1), then (2.3) follows.

We are now ready to state the basic assumptions of our model; they are

$$
\mu(a, P)=\mu(P), \quad \beta(a, P)=\beta_{0}(P) e^{-\alpha a} .
$$

Assumption $(2.4)_{2}$ asserts that the expected number of births is a monotone decreasing function of age (and hence is maximum at age $a=0$ ). Such an assumption is, of course, ludicrous if taken literally, but probably leads to a decent approximation if the population reproduces at a fairly young age. As we shall see, the importance of this model is that it leads to a pair of ordinary differential equations and hence is amenable to analysis. In Sec. 3 we will discuss a model with a more realistic birth law.

We now turn to a derivation of the differential equations. By (2.3) with $g \equiv 1$,

$$
\dot{P}+\mu(P) P-B=0
$$

On the other hand, (2.3) with $g(a)=e^{-\alpha a}$ yields

$$
\dot{G}+\mu(P) G-B+\alpha G=0,
$$

where

$$
G(t)=\int_{0}^{\infty} e^{-\alpha a} \rho(a, t) d a=\frac{B}{\beta_{0}(P)}
$$

Thus (1.1) reduces to the following pair of differential equations: ${ }^{5}$

$$
\begin{aligned}
& \dot{P}=-\mu(P) P+\beta_{0}(P) G \\
& \dot{G}=\left[-\mu(P)+\beta_{0}(P)-\alpha\right] G .
\end{aligned}
$$

[1], Eq. (4.20). There the term $[\beta(P)+\alpha]$ should be $[\beta(P)-\alpha]$. 
The relevant initial conditions follow from $(1.1)_{2,3},(1.2)$, and (2.2); they are

$$
\begin{aligned}
& P(0)=\int_{0}^{\infty} \varphi(a) d a, \\
& G(0)=\int_{0}^{\infty} e^{-\alpha a} \varphi(a) d a .
\end{aligned}
$$

Note that (for $\varphi \neq 0, \alpha>0$ ),

$$
G(0)<P(0)
$$

it is not difficult to show that given any choice of $G(0)$ and $P(0)$ consistent with (2.9), we can find a continuous function $\varphi \geqslant 0$ in $L_{1}(0, \infty)$ such that (2.8) holds.

As mentioned previously, one would generally expect a stronger dependence on $P$ in the survival function $\mu$ than in the maternity function $\beta$. For this reason we consider the further simplification

$$
\beta_{0}(P)=\beta_{0} \quad(=\text { constant }) .
$$

When this is the case we can rewrite (2.7) in terms of $P$ and $B$; the result is

$$
\begin{aligned}
& \dot{P}=-\mu(P) P+B, \\
& \dot{B}=[\gamma-\mu(P)] B,
\end{aligned}
$$

where

$$
\gamma=\beta_{0}-\alpha .
$$

We assume that $\mu$ is class $C^{1}$ and strictly positive. Also, as is natural, we confine our attention to $\mathbb{R}_{+}^{2}=[0, \infty) \times[0, \infty)$, which is an invariant set for (2.11).

\section{PROPOSITION}

The system (2.11) has the following properties:

(a) $\mathcal{E}=\left\{(B, P) \in \mathbb{R}_{+}^{2} \mid B=\gamma P\right\}$ is an invariant set;

$(\beta)$ all equilibrium points lie on $\mathfrak{L}$;

(y) P obeys

$$
\dot{P}=[\gamma-\mu(P)] P \quad \text { on } P \text {. }
$$

Proof. Let $x=B-\gamma P$. Then $\dot{x}=-\mu(P) x$, and it is clear that $x(0)=0$ implies $x(t)=0$ for all $t$. Thus $(\alpha)$ is true. To verify $(\beta)$ note that the 
equilibrium points of (2.11) lie at the origin and at points $(B, P)$ with $\mu(P)=\gamma$ and $B=\mu(P) P$. Thus all equilibrium points lie on $E$. Finally, (2.11) $)_{1}$ reduces to (2.12) when $B=\gamma P$. This completes the proof.

Assume that (2.11) has a closed orbit $\Omega$. Then $\Omega$ must encircle at least one equilibrium point, and hence, by the Proposition, must intersect $E$. But this is not possible. Thus we have the following

\section{THEOREM}

The system (2.11) has no closed orbits.

Another interesting consequence of $(2.10)$ is easily derived as follows. Assume $^{6}$ that $\gamma \neq 0$. By (2.11),

$$
\mu(P)=\frac{B}{P}-\frac{\dot{P}}{P}=\gamma-\frac{\dot{B}}{B},
$$

which implies

$$
\left(\frac{P}{B}\right)^{\cdot}+\gamma\left(\frac{P}{B}\right)=1 ;
$$

hence

$$
\frac{P(t)}{B(t)}=\frac{P(0)}{B(0)} e^{-\gamma t}+\frac{1}{\gamma}\left(1-e^{-\gamma t}\right) .
$$

Thus under the above hypotheses the ratio $P / B$ is independent of the survival function $\mu$.

Next, for any function $f(t)$, let

$$
f^{*}(t)=f(t) \exp \left\{\int_{0}^{t} \mu(P(\alpha)) d \alpha\right\} ;
$$

then (2.11) takes the form

$$
\begin{aligned}
& \dot{P}^{*}=B^{*}, \\
& \dot{B}^{*}=\gamma B^{*},
\end{aligned}
$$

and these equations have the solution

$$
P^{*}(t)=P(0)+B(0)\left[\frac{e^{\gamma t}-1}{\gamma}\right],
$$

\footnotetext{
${ }^{6}$ Analogous results for $\gamma=0$ are obvious.
} 
or equivalently,

$$
P(t) \exp \left\{\int_{0}^{t} \mu(P(\zeta)) d \zeta\right\}=P(0)+B(0)\left[\frac{e^{\gamma t}-1}{\gamma}\right]
$$

Thus the differential equations (2.11) reduce to a single functional equation for the total population $P$. We now solve this equation for a survival function of the form

$$
\mu(P)=b+c P \quad(b>0, \quad c>0)
$$

Indeed, it we substitute (2.15) into (2.14), we find that

$$
\dot{Y}(t) e^{c Y(t)}=\left(P(0)-\frac{B(0)}{\gamma}\right) e^{-b t}+\frac{B(0)}{\gamma} e^{\tau t},
$$

where

$$
\begin{aligned}
Y(t) & =\int_{0}^{t} P(\zeta) d \zeta, \\
\tau & =\gamma-b=\beta_{0}-\alpha-b,
\end{aligned}
$$

and this equation is easily integrated to give

$$
\frac{1}{c}\left(e^{c Y(t)}-1\right)=-\left(P(0)-\frac{B(0)}{\gamma}\right)\left(\frac{e^{-b t}-1}{b}\right)+\frac{B(0)}{\tau}\left(e^{\tau t}-1\right) .
$$

Finally, using (2.16) we solve this equation for $P$ :

$$
\frac{P(t)}{P(0)}=\frac{(1-\xi) e^{-b t}+\xi e^{\tau t}}{1+\eta-\theta l-\eta(1-\xi) e^{-b t}+l e^{\pi t}},
$$

where

$$
\begin{array}{ll}
\xi=\frac{B(0)}{\gamma P(0)}, & \eta=\frac{c P(0)}{b}, \\
l=\frac{c B(0)}{\tau \gamma}, & \theta=\frac{\gamma}{b} .
\end{array}
$$

Clearly,

$$
\begin{aligned}
& P(t) \rightarrow \tau / c \quad \text { for } \quad \tau>0, \\
& P(t) \rightarrow 0 \quad \text { for } \quad \tau<0 .
\end{aligned}
$$

This can also be verified from the phase portrait, which is easily constructed when the assumption (2.15) is satisfied. The result, for $\tau>0$, is shown in Fig.1.

It is interesting to note that, because of (2.15), the differential equation (2.12) reduces to the classical Verhulst equation 


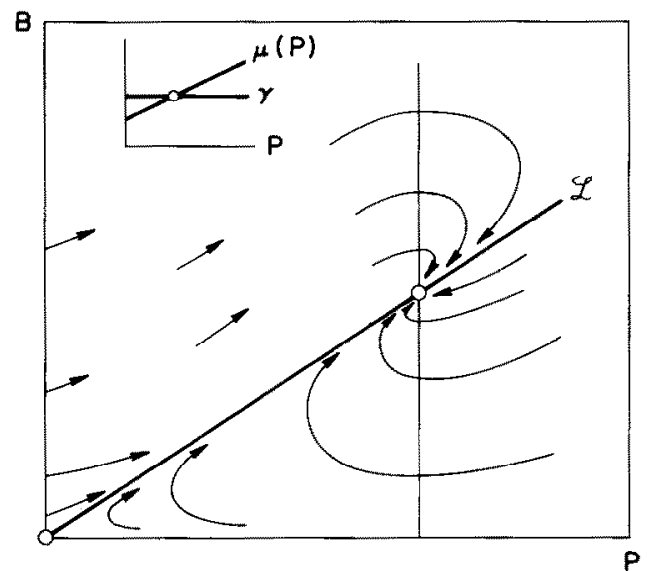

FIg. 1. Phase portrait for survival function $\mu(P)=b+c P$.

$$
\dot{P}+\left(\gamma_{0}-c P\right) P=0
$$

where $\gamma_{0}=\gamma-b=\beta_{0}-\alpha-b$. But (2.12) completely characterizes the population on the invariant set $\mathcal{E}=\{(B, P) \mid B=\gamma P\}$ [cf. ( $\alpha)$ of the Proposition]. Thus our model ${ }^{7}$ exhibits Verhulst behavior whenever the initial data satisfies $B(0)=\gamma \mathrm{P}(0)(\xi=1)$.

Equations (2.13) and (2.17) lead to an expression for the birth rate $B$, and once $P$ and $B$ are known we can use (1.3) to compute the age distribution $\rho(a, t)$ for a particular initial distribution $\varphi(a)$. (This is the first place that the actual shape of the initial distribution plays a role.)

To obtain a better understanding of our model we carried out some numerical calculations for the special case in which

$$
\eta=\frac{1}{2}, \quad \tau=b(>0) .
$$

$P(t) / P(0)$ is graphed in Fig. 2 for various values of $\xi=B(0) / \gamma P(0)$. As remarked above, for $\xi=1$ the population increases monotonically with time as predicted by the Verhulst equation. For $\xi=\frac{1}{2}$ and $\xi=2$, however, the behavior is nonclassical. In the former case the initial birth rate is "small" relative to the initial population, and the population initially decreases. On the other hand, when $\xi=2$ the initial birth rate is "large," and the population surges to a maximum which is larger than its ultimate equilibrium value. This behavior is also apparent from the phase portrait (Fig. 1).

Figure 3 shows the age distribution $\rho(a, t)$ as a function of age for various

\footnotetext{
${ }^{7}$ Under the assumptions (2.4), (2.10), and (2.15).
} 


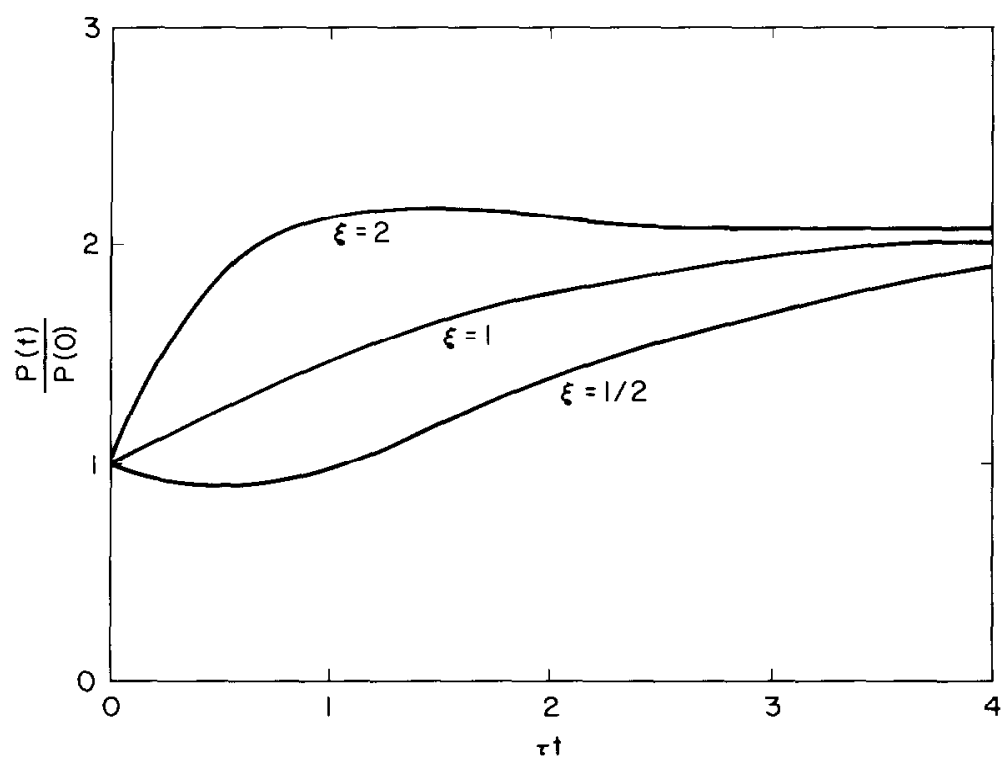

FIG. 2. $P(t) / P(0)$ versus dimensionless time $\pi t$ for various values of $\xi=B(0) / \gamma P(0)$. Here $\eta=\frac{1}{2}$ and $\tau=b$.
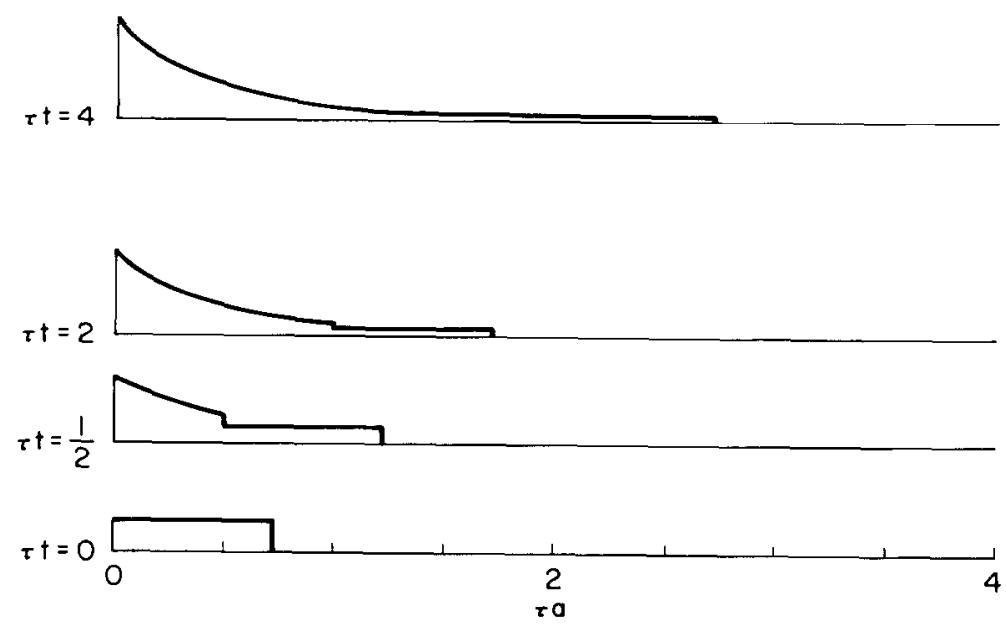

Fig. 3. Dimensionless age distribution $\rho / B_{0}$ as a function of dimensionless age $\tau a$ for various values of dimensionless time $\tau t$. Here $\eta=\frac{1}{2}, \tau=b, \alpha=4 b$, and $\xi=1$. 
times. We performed this calculation for the special case in which

$$
\eta=\frac{1}{2}, \quad \tau=b, \quad \alpha=4 b, \quad \xi=1,
$$

and

$$
\varphi(a)=\left\{\begin{array}{cc}
\varphi_{0}, & 0 \leqslant a \leqslant a_{0} \\
0, & a_{0}<a
\end{array}\right.
$$

Because of (2.18), $\varphi_{0}$ and $a_{0}$ are not arbitrary; in fact, $\varphi_{0} / B_{0}=0.70$ and $\tau a_{0}=0.71$ (approximately). In looking at Fig. 3 one should bear in mind that $\rho$ is a solution of the first-order partial differential equation $(1.1)_{1}$, whose characteristics are straight lines of the form $t=a+$ constant. The data $\varphi$ are inconsistent ${ }^{8}$ in the sense that $\varphi(0) \neq B(0)$, and this inconsistency results in a discontinuity which propagates along the characteristic through $a=0, t=0$. Similarly, the discontinuity in $\varphi$ at $a=a_{0}$ propagates along the characteristic through $a=a_{0}, t=0$. Figure 3 also illustrates the approach with time of $\rho(a, t)$ to its equilibrium value ${ }^{9}$

$$
\frac{\gamma \tau}{c} \exp \left\{-\int_{0}^{a} \mu(\alpha, P) d \alpha\right\}
$$

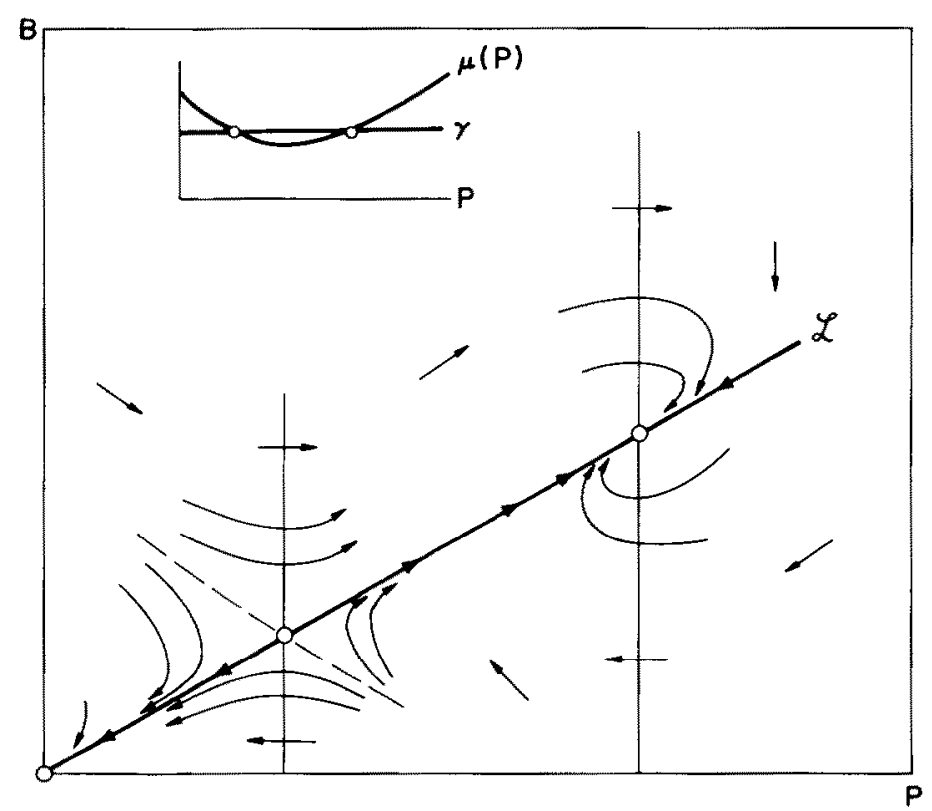

Fig. 4. Phase portrait for more complicated survival function.

${ }^{8} \mathrm{Cf}$. the discussion given on page 285 of [1] in the paragraph preceding Theorem 1.

${ }^{9} \mathrm{Cf}$. [1], Section 3. 
More interesting results are possible for survival functions $\mu(P)$ of a less trivial nature. For example, in certain situations one might expect $\mu(P)$ to be large for $P$ large and also for $P$ small. This motivates our considering a survival function of the form shown in Fig. 4, where the phase portrait is given for the special case in which $\mu(P)=\gamma$ for two values of $P$. As this portrait indicates, the behavior is far more complicated than that corresponding to (2.15) (Fig. 1).

\section{MODEL WITH THREE DIFFERENTIAL EQUATIONS}

As remarked in the last section, the maternity function $(2.4)_{2}$ takes its maximum valuc at age $a=0$, which is unrealistic, as one would expect $\beta(a, P)$ to be zero at $a=0$, increase to a maximum at the age of maximum fertility, and then decrease monotonically to zero. This motivates our studying a model based on the assumptions

$$
\mu(a, P)=\mu(P), \quad \beta(a, P)=\beta_{0}(P) a e^{-\alpha a},
$$

where

$$
\mu(P) \geqslant 0, \quad \beta_{0}(P) \geqslant 0, \quad \alpha>0 .
$$

To derive the corresponding differential equations, note first that (2.5) and (2.6) remain valid in the present circumstances, as they require only that $(3.1)_{1}$ be satisfied. Thus

$$
\begin{aligned}
& \dot{P}=-\mu(P) P+B, \\
& \dot{G}=-\mu(P) G+B-\alpha G,
\end{aligned}
$$

where

$$
G(t)=\int_{0}^{\infty} e^{-\alpha a} \rho(a, t) d a .
$$

Next, if we let

$$
\begin{aligned}
& A(t)=\int_{0}^{\infty} a e^{-\alpha a} \rho(a, t) d a, \\
& g(a)=a e^{-\alpha a},
\end{aligned}
$$

and use the fact that

$$
\int_{0}^{\infty} g^{\prime}(a) \rho(a, t) d a=-\alpha A(t)+G(t)
$$

then (2.3) with $G$ replaced by $A$ implies

$$
\dot{A}=-\mu(P) A-\alpha A+G .
$$


Finally, we note that

$$
B=\beta_{0}(P) A,
$$

and hence (3.3) and (3.4) lead to the system

$$
\begin{aligned}
& \dot{P}=-\mu(P) P+\beta_{0}(P) A, \\
& \dot{G}=-\kappa(P) G+\beta_{0}(P) A, \\
& \dot{A}=-\kappa(P) A+G,
\end{aligned}
$$

where

$$
\kappa(P)=\mu(P)+\alpha .
$$

A thorough study of the system (3.5) is beyond the scope of this paper. We can, however, reach some interesting conclusions when $\beta_{0}(P)$ is independent of $P$, so that (2.10) holds. We begin by rewriting (3.5) as a matrix equation

$$
\dot{x}=-\mu(P) x+M x,
$$

where $x$ is the column vector $(P, G, A)$ and

$$
M=\left(\begin{array}{ccc}
0 & 0 & \beta_{0} \\
0 & -\alpha & \beta_{0} \\
0 & 1 & -\alpha
\end{array}\right) .
$$

This matrix has eigenvalues

$$
\lambda_{1}=0, \quad \lambda_{2}=-\alpha-\sqrt{\beta_{0}}, \quad \lambda_{3}=-\alpha+\sqrt{\beta_{0}} ;
$$

thus there exists a matrix $T$ such that $T A T^{-1}=E$, the diagonal matrix of eigenvalues, and if we let $y=T x$, then

$$
\dot{y}=-\mu(P) y+E y,
$$

which constitutes a diagonal system of equations for $y$. Assume that

$$
\mu(P) \geqslant \mu_{0}>0 .
$$

Then (3.2), (3.6), and (3.7) imply that:

(i) $y_{1}(t)$ and $y_{2}(t)$ tend to zero exponentially as $t \rightarrow \infty$;

(ii) if $\mu_{0}+\alpha>\sqrt{\beta_{0}}$, then $y(t)$ tends to zero exponentially as $t \rightarrow \infty$.

Clearly (i) precludes closed orbits; thus we have the following 


\section{THEOREM}

Under assumptions (2.10), (3.2), and (3.8) the system (3.5) has no closed orbits. Moreover, if $\mu_{0}+\alpha>\sqrt{\beta}_{0}$, then $P(t), G(t), A(t)$, and (hence) $B(t)$ tend to zero exponentially as $t \rightarrow \infty$.

In view of this theorem we conjecture that the general system (3.5) has no closed orbits [under reasonable assumptions concerning the functions $\mu(P)$ and $\left.\beta_{0}(P)\right]$.

The above analysis is easily generalized to the case in which

$$
\mu(a, P)=\mu(P), \quad \beta(a, P)=e^{-\alpha a} \sum_{k=0}^{N} \eta_{k}(P) a^{k} .
$$

Indeed, if we let

$$
G_{k}(t)=\int_{0}^{\infty} a^{k} e^{-\alpha a} \rho(a, t) d a,
$$

then our original system reduces to

$$
\begin{aligned}
& \dot{P}=-\mu(P) P+\sum_{k=0}^{N} \eta_{k}(P) G_{k}, \\
& \dot{G}_{0}=-\mu(P) G_{0}-\alpha G_{0}+\sum_{k=0}^{N} \eta_{k}(P) G_{k}, \\
& \dot{G}_{k}=-\mu(P) G_{k}-\alpha G_{k}+k G_{k-1}
\end{aligned}
$$

$(k=1,2, \ldots, N)$. As before, we assume that

$$
\mu(P) \geqslant \mu_{0}>0, \quad \eta_{k}(P)=\eta_{k}(=\text { constant }),
$$

and rewrite (3.9) as a matrix equation

$$
\dot{x}=-\mu(P) x+M x
$$

with $x$ the column vector $\left(P, G_{0}, G_{1}, \ldots, G_{N}\right)$. We are then led to the following result: If all eigenvalues of $M$ are real and distinct, then (3.9) has no closed orbits; if, in addition, all eigenvalues arc $\leqslant \mu_{0}$, then $x(t) \rightarrow 0$ exponentially as $t \rightarrow \infty$.

Remark. $^{10}$ The function

$$
R(P)=\int_{0}^{\infty} \beta(a, P) \exp \left\{-\int_{0}^{a} \mu(\alpha, P) d \alpha\right\} d a
$$

${ }^{10}$ These comments are due to the referee. 
is called the net reproduction rate. For the system (3.5), augmented by (3.8),

$$
R(P)=\frac{\beta_{0}}{[\alpha+\mu(P)]^{2}} \leqslant \frac{\beta_{0}}{\left[\alpha+\mu_{0}\right]^{2}},
$$

and hence the condition $\mu_{0}+\alpha>\sqrt{\beta_{0}}$ implies

$$
R(P)<1 .
$$

Further, if [in place of (3.8)] we require that $\mu(P)>0, R(P)<1$, then $P(t)$ and $B(t)$ tend to zero as $t \rightarrow \infty$. For the system (3.9),

$$
R(P)=\sum_{k=0}^{\infty} \frac{\eta_{k} k !}{[\alpha+\mu(P)]^{k+1}} \leqslant \sum_{k=0}^{\infty} \frac{\eta_{k} k !}{\left[\alpha+\mu_{0}\right]^{k+1}},
$$

and one is tempted to conjecture that $B(t)$ and $P(t)$ approach zero provided the sum at the extreme right is strictly less than one.

The authors wish to thank K. Pericak for valuable comments. This work was supported by the National Science Foundation.

\section{REFERENCES}

1 M. E. Gurtin and R. C. MacCamy, Non-linear age-dependent population dynamics, Arch. Rational Mech. Anal. 54: 281-300 (1974).

2 F. Hoppensteadt, Mathematical Theories of Populations: Demographics, Genetics and Epidemics, Soc. Ind. Appl. Math., Philadelphia, 1975. 